\title{
Una propuesta curricular para la educación in- tercultural en Honduras: evaluación y resultados
}

\section{A program for the intercultural education in Honduras: evaluation process and its results}

\author{
Delia Fajardo Salinas* \\ dfajardo@upnfm.edu.hn
}

\section{Resumen}

En este artículo se argumenta cómo la literatura de tradición oral, presentada en el formato de libroálbum, puede aprovecharse como un recurso didáctico de gran efectividad en programas de educación intercultural bilingüe, gracias a la identificación de elementos intertextuales entre las narraciones de los pueblos en contacto, y cuya funcionalidad educativa se potencia siguiendo las recomendaciones del enfoque comunicativo, la educación literaria, la literatura comparada, la estética de la recepción y la neurociencia. Para comprobarlo, se evaluó una propuesta curricular diseñada en dichos términos, en centros educativos de la región Norte de Honduras, donde coexisten tres culturas: mestiza, garífuna y miskita. La investigación fue de tipo cualitativa con un diseño de tipo fenomenológico, abierto y emergente. Como resultado principal, se logró una amplia aceptación de esta propuesta por parte de la comunidad educativa, implicando con ello una nueva valoración de la literatura de tradicional oral y de la metodología para enseñar interculturalidad.

Esta investigación fue financiada por el Instituto de Investigación y Evaluación Educativas y Sociales de la Universidad Pedagógica Nacional Francisco Morazán

Docente del Departamento de Letras y Lenguas. Carrera Enseñanza del Español, Universidad Pedagógica Nacional Francisco Morazán, Honduras.

Recibido 05 de mayo de 2018 / Aceptado 06 de junio de 2018. 
Delia Fajardo Salinas

Palabras clave: tradición oral, libro álbum, educación intercultural bilingüe, formación docente en EIB

\begin{abstract}
:
This article argues how the literature of oral tradition, presented as picture book, can be used as a highly effective didactic resource in bilingual intercultural education programs, thanks to the identification of intertextual elements among the narrations of the cultures in contact, and whose educational functionality is enhanced following recommendations of: communicative approach, literary education, comparative literature, the aesthetics of reception and neuroscience. To verify this, a curricu-

lar proposal designed in these terms was evaluated in educational centers in the Northern region of Honduras, where three cultures coexist: mestiza, garífuna and miskita. The research was of a qualitative type with a phenomenological, open and emergent design. As a main result, a wide acceptance of this proposal by the educational community was achieved, implying a new assessment of the traditional oral literature and the methodology to teach interculturality.
\end{abstract}

Keywords: oral tradition, picture book, bilingual intercultural education, teaching training on EIB

\title{
Introducción
}

Según la proyección de población del Instituto Nacional de Estadística, Honduras reúne aproximadamente 9,012,229 de habitantes (INE, 2018) de los cuales entre 10 y 15 por ciento corresponde a nueve pueblos étnicos minoritarios ubicados en diferentes zonas del país: lencas, tolupanes, chortís, pech, tawahkas, negros garífunas, negros isleños, miskitos y nahuas.

En el pasado, la oferta educativa para estos pueblos era únicamente en el idioma oficial, español, y sobre contenidos de la cultura oficial

58 Instituto de Investigación y Evaluación Educativas y Sociales / Universidad Pedagógica Nacional Francisco Morazán

Los artículos de la Revista Electrónica Paradigma del Instituto de Investigación y Evaluación Educativas y sociales de la Universidad (i) $-\left(\begin{array}{l}\text { Pedagóica Nacional Francisco Moraźn, se comparten bajo términos de la licencia Creative Commonss: se permite que otros puedan } \\ \text { descargar las obras y compartirlas con otras personas, siempre y cuando se reconozca su autoría, pero no se pueden cambiar de }\end{array}\right.$ hinguna manera ni se pueden utilizar comercialmente. 
hegemónica, pero desde hace unas dos décadas esta situación ha venido cambiando. Actualmente, existen centros de educación pública especializados en EIB en todos los territorios de los pueblos étnicos mencionados, y cuentan con docentes egresados de los programas también especializados, y con recursos especialmente diseñados para cada cultura en la lengua de cada pueblo: libros de texto, libros de lectura, diccionarios y gramáticas.

A pesar de ello, persisten un amplio abanico de carencias que impiden la óptima ejecución de la EIB en las aulas hondureñas, sobre todo en aquellas con predominio de población mestiza, donde se suelen reproducir sutil y naturalmente políticas y conductas antiinterculturales. Contar con recursos didácticos y orientaciones metodológicas para que los docentes en servicio (mestizos), entiendan y apliquen la pedagogía de la educación intercultural es una de esas carencias.

Si bien el currículo oficial indica la enseñanza de la interculturalidad, y sugiere algunas estrategias, ello no asegura que todo el personal docente, a nivel nacional, sepa realizarlas adecuadamente por su falta de preparación en la teoría de la pedagogía intercultural. Además, consideramos que dicho diseño curricular adolece de una perspectiva limitada sobre cómo enseñar la interculturalidad ya que se presenta como contenido puntual, incluso hasta esporádico, en lugar de constituirse en eje transversal que impregne toda la formación, todas las áreas curriculares, toda la vida escolar.

Este panorama fue diagnosticado mediante una investigación de campo en el año 2006, en el marco de nuestro trabajo de Tesis Doctoral (Fajardo, 2010) y sirvió como motivación y justificación para el diseño de una propuesta curricular que ofreciera soluciones ante esas debilidades. La preparación de esta propuesta se culminó en el año 2009, y en 2016 logramos llevar a cabo su evaluación en centros educativos de la costa Norte de Honduras, caracterizada por su diversidad cultural con la presencia de mestizos, negros garífunas, miskitos, y en menor cantidad: negros ingleses, pech, tawahkas e inmigración extranjera. 
En ese artículo presentamos los resultados de este último proceso. En primer lugar, exponemos los principales planteamientos teóricos que fundamentan la propuesta curricular diseñada y una breve descripción de la misma. En segundo lugar, se explican los procedimientos metodológicos; en tercer lugar, se presenta un resumen de los resultados obtenidos.

\section{Fundamentación Teórica}

Ante la problemática descrita, nuestro interés se centró en diseñar una propuesta curricular cuyo recurso central fuera una colección de cuentos tomados de la tradición oral, porque las narraciones ocupan un lugar protagónico en la transmisión cultural y formación identitaria durante la infancia, e inciden directa e indefectiblemente en su desarrollo cognitivo, como lo demuestran múltiples estudios desde diversas disciplinas (Adam \& Lorda, 1999; Bettelheim, 1974, 1999; Brasey \& Debailleul, 2004; Colomer, 1998, 1999, 2002; Fernández, 2000; Janer, 2010; Núñez, 2005; Vigotsky, 1986).

En relación específicamente al valor educativo de la tradición oral, Pilar Núñez argumenta sobre su utilidad ancestral para integrar al infante en unas coordenadas culturales y para que valore dicho legado y crítica a la institución escolar por descuidar esta función de procurar "la socialización y la inserción del niño en su cultura por medio de la valoración de sus tradiciones" (2005: 193). Por lo tanto, propone que se rescate y sea aprovechada en el marco de los nuevos enfoques de la educación literaria y el enfoque comunicativo, tomando en cuenta sus transformaciones en su proceso de adaptación a la modernidad por el contacto con el mundo letrado y tecnológico, el cual sin lugar a dudas ha modificado por ejemplo sus formatos y causes de transmisión.

Núñez señala además que la literatura de tradición oral se ha convertido en un potente intertexto temático y discursivo. Esta aseveración fue confirmada en el contexto multicultural de Honduras mediante el análisis de 216 relatos, entre los cuales se logró identificar temas y personajes comunes de acuerdo a una tipología generada de un análisis literario comparado, realizado en el marco 
de la Tesis Doctoral ya aludida y que se puede consultar en otra publicación de la autora (Fajardo, 2014) en la cual argumentamos el porqué utilizar el formato de libro álbum. Con base en el mencionado análisis fue posible seleccionar una muestra representativa de 16 relatos para conformar un canon formativo (Mendoza \& Cantero, 2003) intercultural hondureño, dirigido a educar en los principios de la interculturalidad. Entre estos, cabe enumerar los siguientes:

- El reconocimiento del Otro como interlocutor en igualdad

- Conocer las otras culturas distintas a la propia

- Valorar la diversidad cultural en los lindes de la nación

- Superar la desconfianza, temores, prejuicios, estereotipos

- Aspirar a un aprendizaje mutuo, con la voluntad para hacerlo

- Procurar una comunicación efectiva

- Optar por el trabajo conjunto y efectivo en la organización de la vida social, en función sobre todo, de perfilar un proyecto válido y consensuado de reconfiguración del Estado-nación. (Duarte, 1999: 25)

Los cuentos de tradición oral en formato de libro álbum, se insertaron como recursos nucleares en el entramado de una propuesta curricular que se diseñó siguiendo los planteamientos teóricos y metodológicos del enfoque comunicativo y la educación literaria (Mendoza, 1998, 1999; Sánchez, 2003), la pedagogía intercultural (Aguado, 2003) la literatura comparada (Bordons \& Díaz-Plaja, 2004) y la estética de la recepción (Jauss, 1976). En síntesis, consta de un folleto para la capacitación docente, un guion metodológico general, la selección de 16 cuentos de tradición oral y las guías didácticas para cada uno. Estos cuentos se agrupan por ciclo, correspondiendo cuatro a cada uno (ver Tabla 1):

\section{Tabla 1}

Asignación del canon formativo intercultural por ciclo

\begin{tabular}{clc}
\hline \multicolumn{1}{c}{ Cuento } & Pueblo \\
\hline \multirow{3}{*}{ Prebásica } & El palo de brujo & Lenca \\
& Anasi, el conejo y compadre tigre & Isleño \\
& La joven prudente & Miskito \\
& Barugu & Garífuna \\
\hline
\end{tabular}


Delia Fajardo Salinas

\begin{tabular}{clc}
\hline & La tierra se queja con Toman & Tolupán \\
Básica Ciclo I & Juan y el conejo & Miskito \\
& El pacto que no se realizó & Lenca \\
& La leyenda de Chawuawuaca & Pech \\
& El Dingutídiuma & Garífuna \\
Básica Ciclo II & El venado y su compadre tigre & Tawahka \\
& La siguamonta & Chortí \\
& El sisimite, la niña y el caballito & Tawahka \\
& La sirena de Botija & Garífuna \\
Básica Ciclo III & El origen del Pech & Pech \\
& La danta que hizo Dugú & Garífuna \\
& El origen de los ríos & Tolupán \\
\hline
\end{tabular}

Todas las guías didácticas siguen una misma secuencia:

1. Una actividad de animación que puede ser un juego o la confección de una manualidad, o ambos, u otro tipo de actividad lúdica.

2. Narración oral del cuento con la lectura visual del libro álbum, con prácticas propias de comprensión lectora: preguntas de anticipación y predicción sobre el inicio, el final, el futuro, etc.

3. Ejercicios de análisis y/o reflexión en función del estándar del currículo oficial y de los indicadores de la competencia intercultural asignados. Por ejemplo: inducción a la empatía (ponerse en los zapatos del otro, conectar con la experiencia propia); ejercicios de comparación/reconocimiento de diferencias y similitudes.

4. Actividad plástica: crear un objeto simbólico que quede vinculado a los aprendizajes, a las ideas compartidas, a la memoria de las experiencias con el libro álbum.

5. Ejercicios de creación y manipulación literaria como crear, cambiar o mezclar: el título, el inicio, el final, personajes, roles, acontecimientos, lugares, época.

6. Actividades de animación para recordar el relato cuando se inicia una nueva sesión para darle continuidad a la guía didáctica.

7. Actividades alternativas y/o complementarias: escritura en

Instituto de Investigación y Evaluación Educativas y Sociales / Universidad Pedagógica Nacional Francisco Morazán

Los artículos de la Revista Electrónica Paradigma del Instituto de Investigación y Evaluación Educativas y Sociales de la Universidad Pedagógica Nacional Francisco Morazán, se comparten bajo términos de la Licencia Creative Commons: Se permite que otros puedan inguna manera ni se pueden utilizar comercialmente. 
L1 y/o L2, dramatización, investigación de contenidos culturales aludidos en los cuentos, manualidades, teatro con títeres, etc.

Esta selección de actividades responde a las recomendaciones de las teorías ya aludidas. Y además, de la neurociencia ya que procuran un ambiente lúdico que active emociones favorables para el aprendizaje y la memoria de largo plazo (Mora, 2017; Casafont y Casas, 2017). Los neurocientíficos proponen que al enseñar, debe haber novedad y sorpresa, juego, actividad artística o creativa, movimiento, para generar emociones positivas (interés, curiosidad) que dirijan una atención sostenida, y también un vínculo afectivo que genere empatía para beneficiar el desarrollo del cerebro social considerando su naturaleza plástica (Bueno, 2016; 2018). Todo ello está presente en los elementos y actividades de nuestra propuesta curricular.

\section{Metodología}

En relación al encuadre metodológico, ha sido cualitativo con un diseño de tipo fenomenológico, abierto y emergente (Hernández, Fernández \& Baptista, 2014). La investigación se llevó a cabo en el Departamento de Atlántida. Se inició en febrero y concluyó en octubre, de 2016. El muestreo fue de tipo no probabilístico, por conveniencia (Ibíd., 390).

La muestra final quedó conformado por un total de 404 niñas y niños, en su mayoría mestizas y mestizos, pero con presencia de minorías (garífunas y miskitus, o a la inversa). Esta configuración multicultural fue ideal para evaluar la propuesta, ya que buscamos precisamente este tipo de grupos donde hubiera algún tipo de minoría, para observar el impacto de las enseñanzas interculturales en las relaciones sociales entre los estudiantes, y en la autopercepción de esos que eran minoría.

Cabe mencionar que las personas reaccionaban con extrañeza sobre nuestro interés en realizar el proyecto de evaluación en centros 
educativo que no eran EIB, o sea, que no eran solo de garífunas o solo de miskitos. Esto demostraba la persistencia de un entendimiento equivocado de la interculturalidad, es decir como educación diferenciada o especial solo para indígenas y afrodescendientes, y confirmaba una vez más, las motivaciones originales y la justificación del proyecto hacia la promoción masiva de un concepto de interculturalidad, de acuerdo a los principios que hemos mencionado antes, en todo el Sistema Educativo Nacional, para toda la población, precisamente para corregir ese imaginario erróneo que mantiene a esta población en una posición simbólica marginal.

Una vez configurada la muestra, se inició con la capacitación de las docentes participantes y después se procedió a organizar un horario de acompañamiento que permitiera una visita a la semana, para que la frecuencia de las actividades a realizar fuera semanal, o al menos quincenal, tal como lo sugiere el guión metodológico de la propuesta. Este período de trabajo abarcó cuatro meses (de mayo a agosto), con el fin de desarrollar la guía didáctica completa de un cuento cada mes.

Para finalizar este apartado, destacamos que la pregunta de investigación central de nuestro estudio ha sido la siguiente: ¿en qué medida la literatura de tradición oral de los pueblos indígenas y afro hondureños es un recurso de gran potencial didáctico para cumplir con los propósitos de la educación intercultural, cuando se reelabora en formato de libro álbum y se inserta en una propuesta curricular que aplica las recomendaciones metodológicas de la educación literaria, el enfoque comunicativo, la literatura comparada, la estética de la recepción y la neurociencia?

Procedimientos de recolección de datos y análisis

Las sesiones de cada actividad en las escuelas fueron grabadas, y este registro servía de apoyo para el posterior llenado de un diario de campo. En total, se acumularon alrededor de 112 vídeos y una cantidad abundante de fotografías, o sea que contamos con un registro de las reacciones de niñas y niños a los elementos de la propuesta curricular, donde se puede verificar su nivel de impacto (en cuanto a

64 Instituto de Investigación y Evaluación Educativas y Sociales / Universidad Pedagógica Nacional Francisco Morazán

(i) 1 Los artículos de la Revista Electrónica Paradigma del Instituto de Investigación y Evaluación Educativas y Sociales de la Universidad (1) - -1 Pedagógica Nacional Francisco Morazán, se comparten bajo términos de la Licencia Creative Commons: Se permite que otros puedan ninguna manera ni se pueden utilizar comercialmente. 
deseo de participar, nivel de atención, estado anímico, cumplimiento de las tareas, interés en el material didáctico, etc.). Aunque debido a limitaciones de tipo técnico, no siempre fue posible grabar las reacciones o actitudes de todos los niños y las niñas en el aula, en todos los grupos participantes.

Al finalizar cada sesión de trabajo en aula, se dedicaban unos minutos para entrevistar a la docente con el fin de evaluar lo realizado. Esta conversación informal fue grabada y también sirvió de apoyo para el posterior llenado del diario de campo, con la transcripción de fragmentos que se convirtieron en unidades o segmentos de significado relevante, efectuándose así el primer proceso de codificación abierta, ya que se fueron generando categorías de análisis tentativas; de esta manera, el diario evolucionó de una narrativa lineal cronológica a una clasificación de los segmentos de significado, provenientes de las entrevistas y la observación participante en esas categorías, que se fueron afinando, hasta que no surgieron nuevas, logrando así la saturación. El criterio para identificar los segmentos o unidades de análisis fue de "libre flujo", es decir, sin establecer una medida restrictiva o específica para su extensión (Hernández et al., 2014, p. 427). Como producto de este análisis de los datos emergentes, el diario de campo se organizó en torno a tres unidades: las docentes, las niñas y los niños, los cuentos ilustrados. Cada uno con categorías de análisis diferentes pero complementarios.

En cuanto al tipo de categorías manejadas, en su mayoría fueron "esperadas" y "centrales para el planteamiento del problema", es decir, sirven para responder nuestra pregunta central de investigación; algunas pocas fueron "inesperadas" y aportaron hallazgos no previstos, y con ello importantes recomendaciones para futuras réplicas. Debido a que se trata de una evaluación, y siguiendo la clasificación de Bogdan y Biklen (2003) (en: ibíd., 433), reunimos categorías sobre todo de la "perspectiva de los participantes" de la comunidad educativa, así como sobre: actividades, estrategias, relaciones, y ambiente.

Otro procedimiento para la recolección de importantes datos fueron las reuniones de evaluación final con las docentes, organizadas en 
tres grupos focales por zona, y que se realizaron en septiembre. Se utilizó como instrumento una hoja de llenado individual, con una lista de categorías de tipo teórico (ibíd.). También se realizaron entrevistas durante el mes de septiembre y octubre, en la etapa final de la investigación, para recabar las impresiones y valoraciones de diversos actores de la comunidad educativa: estudiantes, madres de familia, personal directivo y colaboradoras (traductoras o cuentacuentos invitadas).

En general, el análisis de datos consistió en un proceso de clasificación mediante la técnica de la metacodificación (Hernández et. al., 2014: 440): buscar en qué categoría encajaba cada segmento en tanto representativo de la misma, y luego en dónde encajaba cada categoría en tanto evidencia de un resultado esperado, es decir, su valencia fue establecida de acuerdo a los principales objetivos del proyecto de investigación diseñado para evaluar la propuesta curricular. Para el análisis de los datos también se realizaron procesos de triangulación, vinculando la opinión de los distintos participantes: docentes, estudiantes, otros actores, investigadora principal, en torno a esas categorías de análisis que resultaban complementarias, por ejemplo: utilidad de los cuentos de tradición oral para la educación intercultural, calidad de los libros álbum, calidad de las guías didácticas, nivel de aprendizaje de la interculturalidad, entre otras.

\section{Resultados}

Con base en estos análisis, podemos asegurar que todos los grupos escolares participantes demostraron una recepción altamente positiva de las enseñanzas interculturales y alta motivación a participar en las actividades, excepto por grupos en edad adolescente, quienes quizás ya han perdido su capacidad de asombro ante un entorno social que se revela desmitificado, conflictivo y violento; quizás en estos grupos se ha afirmado ya un código lingüístico restringido (Bernstein, 1977) pero faltarían más indagaciones para asegurarlo. Del total de la muestra, estamos hablando del grupo de noveno grado, con 23 estudiantes, con sobre edad, es decir, algunos eran mayores a la edad correspondiente al grado. Y también del grupo de séptimo

66 Instituto de Investigación y Evaluación Educativas y Sociales / Universidad Pedagógica Nacional Francisco Morazán

(i) 1 Los artículos de la Revista Electrónica Paradigma del Instituto de Investigación y Evaluación Educativas y Sociales de la Universidad (i) (-) -) Pedagógica Nacional Francisco Morazán, se comparten bajo términos de la Licencia Creative Commons: Se permite que otros puedan EY NC ND descargar las obras y compartirlas con otras personas, 
grado, con 32 estudiantes, donde se pudo percibir un entusiasmo menor. Esto invita a replantear el diseño de actividades dirigidas a estas edades, para que vayan más acorde a sus capacidades e intereses.

En general, el alumnado respondía sin mayor dificultad a las preguntas didácticas estipuladas en las guías y se lograba que siguieran el proceso inductivo de construcción de los aprendizajes interculturales meta. Destaca como dato muy valioso que ya para finalizar el período de trabajo de campo, varios estudiantes de los grupos de quinto y sexto grado (a los inicios muy callados), habían empezado a involucrarse con entusiasmo y confianza, mostraban iniciativa y deseo de participar.

Para conocer el nivel de aprendizaje de la interculturalidad como concepto, hicimos la pregunta: ¿A qué te suena la interculturalidad o lo intercultural? Y comprobamos que esta palabra quedó vinculada a una memoria afectiva feliz, como lo demuestran las siguientes respuestas: "a jugar", “diversión”, "me acuerdo de usted”. Este último segmento de significado fue dicho con una enorme sonrisa por parte del niño entrevistado. $Y$ podemos agregar como dato que varios grupos de niños (de prebásica y primer ciclo), siempre que retornábamos al centro educativo para realizar alguna diligencia puntual, se lanzaban a saludarnos con cariño en abrazo colectivo y/ o nos decían los saludos que les habíamos enseñado en las lenguas indígenas, como buitibinafi (buenos días en lengua garífuna), semanas después de haber finalizado nuestra presencia en aulas.

En este dato subyace una gran evidencia de logro si tomamos en cuenta las más recientes a portaciones de la neurociencia. Por ejemplo, se ha planteado que el cerebro es un órgano de conocimiento emocional, y que los niños no aprenden contenidos, aprenden al maestro, es decir, aprenden de él mediante el vínculo. En palabras de Casafont y Casas: "Tiene más fuerza el ejemplo que las reglas" (2017: 147). De forma más extensa, señalan que: "El alumno aprende por imitación, atención compartida y empatía [...] Somos su ejemplo." (Íbid., 159) Esto es así porque en la capacidad de aprendizaje del cerebro participan "las neuronas espejo y las neuronas bipolares Von 
Economo relacionadas con la capacidad empática y el cerebro social. [...] ambos tipos de neuronas intervienen en la atención, conducta social y ética, la emoción y la cognición." (Íbid., 76)

Por tanto, dado que nuestra interacción comunicativa dejó un impacto emocional positivo en los grupos de alumnos y docentes, creemos que podemos darnos por satisfechas, ya que de eso se trataba, de construir un vínculo emocional que favoreciera la activación de esas neuronas, espejo y bipolares, para aprender una conducta social y ética de tipo intercultural. Y lo hicieron al experimentar una forma positiva de relacionarse con la alteridad a través de actividades lúdicas y artísticas, en contacto con diversidad de cuentos de tradición oral que mostraban puntos en común entre los pueblos y comprobando que también consigo mismos, y que los llevó a la conclusión: no somos tan diferentes.

De esta manera se favoreció la creación de nuevas conexiones sinápticas en el cerebro propias de una manera de ser intercultural, ya que estuvieron presentes los ingredientes básicos para lograr un aprendizaje perdurable, desde los principios de la neurociencia que ya señalamos antes. Sin duda, la repetición debe perdurar para que dichas conexiones sinápticas se sostengan, por ello es que la propuesta curricular está diseñada para que cada año los estudiantes vuelvan a vivenciar lo mismo pero con diferentes cuentos, durante toda su escolarización. Se trata de formar cerebros interculturales en lugar de cerebros monoculturales.

Deseamos destacar dos testimonios muy reveladores del sutil pero valioso impacto de la propuesta curricular para generar aprendizajes de tipo intercultural. Estos testimonios tienen que ver con niños que constituían una minoría étnica en el aula. En las entrevistas realizadas tanto a sus maestras de grado como a ellos mismos, pudimos comprobar que la experiencia había marcado un antes y un después en su autoconcepto y en sus relaciones sociales, con un efecto altamente positivo en su autoestima. El más joven de estos niños (mulato, con apenas 10 años de edad) declaró ante la clase, de manera casual, que lamentaba que su madre garífuna no hablara la lengua de su pueblo, ya que así él sería bilingüe, según relató su maestra en la reunión de grupo focal. Ella añadió que: 
Él antes me decía: profe no quiero asolearme mucho porque no quiero ser así trigueño [oscuro]... y así algunas cositas... Pero ya después de que hicimos todo esto, ahora él dice ahí en el aula " ${ }^{\prime} M e$ siento orgulloso de ser mulato!"

Sin duda este testimonio demuestra que la propuesta curricular es capaz de lograr sus objetivos, de provocar aprendizajes interculturales que preparan mejor a niños y niñas para entablar óptimas relaciones sociales de convivencia y vínculos afectivos saludables porque “...cuanto mejor sea el autoconcepto del niño, mejor serán las relaciones que establecerá" (Casafont y Casas, 2017: 162).

El relato de otra maestra sobre la evolución de un niño garífuna también resulta muy ilustrativo sobre este impacto altamente positivo:
Él al principio no quería participar en nada... o él sabía cuando era [la actividad de cuentacuentos] y no [llegaba a clase]... y ahorita en los desfiles, hasta salió en los grupos étnicos, con un traje, un tambor y todo... y todo mundo asustado porque a él nunca le gustaba integrarse así... y le tomaban fotos y más se ponía a tocar el tambor...

Es decir, el niño evolucionó del rechazo a que su diferencia cultural destacara hacia la integración y aceptación, de no querer ser visto en su diferencia cultural a sentirse orgulloso de su identidad.

Aunque se trate de solamente dos casos, demuestran que sí existe una problemática de discriminación callada, que esta investigación sacó a la luz, poniendo en entredicho los discursos políticamente correctos que suelen circular en los centros educativos pero que suelen ignorar el sentir real de muchos niños y niñas, que prefieren pasar desapercibidos antes que ser reconocidos en su diferencia, dado el bajo valor simbólico que posee su cultura original en el contexto social hondureño. Los datos cualitativos recolectados mediante este proyecto de investigación demuestran que sí es posible cambiar ese escenario. 


\section{Conclusión}

Esperamos que esta breve discusión de resultados, que apenas representa un fragmento de un informe mucho más amplio y detallado, sea lo suficientemente ilustrativa para fundamentar la respuesta afirmativa que podemos dar a nuestra pregunta central de investigación. Significa que la conclusión principal de este estudio es, que la literatura de tradición oral de los pueblos indígenas y afrodescendientes sí es un recurso de gran potencial didáctico para cumplir con los propósitos de la educación intercultural, cuando se reelabora en formato de libro álbum y se inserta en una propuesta curricular que aplica las recomendaciones teórico-metodológicas de la educación literaria, el enfoque comunicativo, la literatura comparada, la estética de la recepción y en coherencia con los principios de la neurociencia.

Esto fue confirmado por un grupo de docentes en servicio, con muchos años de experiencia, quienes cambiaron su antigua visión sobre esos cuentos tradicionales (por una nueva valoración muy positiva), y su imaginario erróneo sobre el concepto de interculturalidad, entiendo ahora que "la interculturalidad somos todos", en sus propias palabras. Su grado de aceptación de la propuesta curricular en su conjunto fue tan alto, que propusieron integrarla a su jornalización del siguiente año desde el inicio del periodo escolar, y para todos los grados, es decir, involucrando a toda la escuela, mientras que desde la Dirección Departamental de Atlántida se esperaba promoverla en otros centros educativos.

\section{Referencias}

Adam, J. \& Lorda, C. (1999). Lingüística de los textos narrativos. Barcelona: Ariel.

Aguado, T. (2003). Pedagogía intercultural. Madrid: McGraw-Hill.

Bernstein, B. (1977). Class, Codes and Control, vol. 3. London: RKP.

70 Instituto de Investigación y Evaluación Educativas y Sociales / Universidad Pedagógica Nacional Francisco Morazán

Los artículos de la Revista Electrónica Paradigma del Instituto de Investigación y Evaluación Educativas y Sociales de la Universidad Pedagógica Nacional Francisco Morazán, se comparten bajo términos de la Licencia Creative Commons: Se permite que otros puedan
descargar las obras y compartirlas con otras personas, siempre y cuando se reconozca su autoría, pero no se pueden cambiar de ninguna manera ni se pueden utilizar comercialmente. 
Bettelheim, B. (1974). Heridas simbólicas. Los ritos de la pubertad y el macho envidioso. Barcelona: Barral.

Bettelheim, B. (1999). Psicoanálisis de los cuentos de hadas. Barcelona: Crítica.

Bordons, G; \& Díaz-Plaja, A. (2004). Les aportacions de la teoria de la literatura a l'ensenyament. En G. Bordons y A. Díaz-Plaja (coords.), Ensenyar literatura a secundària. La formació de lectors crítics, motivats i cultes (pp. 7-16). Barcelona: Graó

Brasey, E; \& Debailleul, J-P. (2004). Vivir la magia de los cuentos. El poder sanador de los cuentos tradicionales. Cómo lo maravilloso puede sanar nuestras vidas (5ta ed.). Madrid: EDAF

Bueno i Torrens, D. (2016). Cerebroflexia. El arte de construir el cerebro. Barcelona: Plataforma.

Bueno i Torrens, D. (2018). Cómo cambia nuestro cerebro al aprender. BBVA Aprendemos juntos. Recuperado de: https:// aprendemosjuntos.elpais.com/especial/la-inteligenciaviene-de-serie-o-se-entrena-david-bueno/v/la-inteligencia-vienede-serie-o-se-entrena-c/

Casafont, R; \& Casas, L. (2017). Educarnos para educar. Neuroaprendizaje para transformar la educación. Barcelona: Paidós.

Colomer, T. (1998). La formación del lector literario: narrativa infantil y juvenil actual. Madrid: Fundación Germán Sánchez Ruipérez.

Colomer, T. (1999). Introducción a la literatura Infantil y juvenil. Madrid: Síntesis.

Colomer, T. (coord.) (2002). Siete llaves para valorar las historias infantiles. Madrid: Fundación Germán Sánchez Ruipérez, 20051ª reimp. 
Duarte Méndez, Sergio C. (coord.) (1999). Guía Sobre Interculturalidad. Primera Parte. Fundamentos Conceptuales. Guatemala: Proyecto $\mathrm{Q}^{\prime}$ Anil / PNUD.

Fajardo, D. (2010). La Educación Intercultural Bilingüe (EIB) en Honduras: teoría y didáctica para la adquisición de la competencia intercultural. (Tesis inédita de doctorado). Universidad de Barcelona, España.

Fajardo, D. (abril-junio, 2014). El potencial didáctico del libro-álbum para la educación literaria-intercultural. Educar em Revista (52). Recuperado de http://ojs.c3sl.ufpr.br/ojs2/index.php/educar/ article/view/36609/22631

Fernández Paz, A. (2000). Estrategias para contar cuentos y escribir relatos en la escuela primaria. Textos de Didáctica de la Lengua y la Literatura (25), 23-33

Hernández, R., Fernández; C. \& Baptista, M. (2014). Metodología de la investigación (6ta ed.). México: McGraw-Hill.

Instituto Nacional de Estadística. (2018). Proyecciones de población. Proyección 2014-2030. Recuperado de: http://170.238.108.227/ binhnd/RpWebEngine.exe/Portal?BASE=PROYPOB\&lang=ESP

Janer, G. (2010). Literatura oral y ecología de lo imaginario. Madrid: Fundación Germán Sánchez Ruipérez.

Jauss, H. R. (1976). La literatura como provocación. Barcelona: Península.

Mendoza, A. (1998). El proceso de recepción lectora. En A. Mendoza (Coord.): Conceptos clave en Didáctica de la Lengua y la Literatura (pp. 169-189). Barcelona: SEDLL / ICE Universitat de Barcelona / HORSORI

Mendoza, A. (1999). Educación literaria y formación plurilingüe. En M. García et al. (Eds.), Ensenyament de Llengües i Plurilingüisme. I

Instituto de Investigación y Evaluación Educativas y Sociales / Universidad Pedagógica Nacional Francisco Morazán

Los artículos de la Revista Electrónica Paradigma del Instituto de Investigación y Evaluación Educativas y Sociales de la Universidad Pedagógica Nacional Francisco Morazán, se comparten bajo términos de la Licencia Creative Commons: Se permite que otros puedan pueden cambiar de 
Congreso Internacional de Didáctica de Lengua y Literatura (pp. 97114). Valencia: Universidad de Valencia

Mendoza, A. \& Cantero, F. (2003). El canon formativo y la educación lecto-literaria. En A. Mendoza (Coord.), Didáctica de la Lengua y la Literatura (pp. 349-378). Madrid: Pearson Educación.

Mora Teruel, F. (2017). Neuroeducación: solo se puede aprender aquello que se ama. $2^{\mathrm{a}}$ ed. Madrid: Alianza.

Núñez, D., M. P. (2005). Literatura de tradición oral y nuevas tecnologías en la educación literaria escolar. En C. Gonzáles et al. (Coords.). Leer de nuevo, leer lo nuevo (pp. 191-200). Barcelona: Grupo Editorial Universitario.

Sánchez, L. (2003). De la competencia literaria al proceso educativo: actividades y recursos. En A. Mendoza (Coord.), Didáctica de la Lengua y la Literatura (pp. 319-348). Madrid: Pearson Educación.

Vigotsky, L. (1986). La imaginación y el arte en la infancia. $6^{\mathrm{a}}$ ed. Madrid: Akal, 2006.

Vigotsky, L. (1986). Thought and Language. Boston: The Massachussetts Institute of Technology. Trad. Esp. (1995): Pensamiento y lenguaje. Barcelona: Paidós. 

BY NC ND ninguna manera ni se pueden utilizar comercialmente. 